\title{
ARTIGOS
}

\section{EL PAPEL DE LA INSPECCIÓN EDUCATIVA EN LOS PROCESOS DE CAMBIO}

HÉCTOR MONARCA • NOELIA FERNÁNDEZ-GONZÁLEZ

\section{RESUMEN}

Se presentan los resultados de una investigación cuyo objetivo ha sido analizar los procesos relacionados con la implementación de una innovación educativa promovida por la Ley Orgánica de Educación en España: la incorporación de las competencias básicas a las prácticas educativas de los centros. En esta ocasión se pone el acento en el papel que ha desempeñado la inspección educativa en dicho proceso. La investigación se desarrolló mediante una metodología cualitativa, realizando un estudio de casos múltiples en seis centros educativos de tres comunidades autónomas diferentes. En los casos abordados, los resultados evidencian que el papel desplegado por los inspectores educativos representa, con mucha frecuencia, un rasgo obstaculizador de las innovaciones propuestas, el cual viene dado, principalmente, por una excesiva vigilancia de los documentos de los centros educativos. En este sentido, las conclusiones invitan a una reflexión profunda sobre el papel que estos actores educativos deben asumir en el desarrollo de las políticas públicas, junto con la necesidad de contemplar un rol más centrado en el asesoramiento.

POLÍTICAS DE EDUCACIÓN • INSPECCIÓN DE ESCUELAS • ELABORACIÓN DEL PLAN DE ESTUDIOS • INNOVACIONES EDUCACIONALES

\section{THE ROLE OF SCHOOL INSPECTION IN THE PROCESSES OF EDUCATIONAL CHANGE}

\section{ABSTRACT}

This article presents the results of a study whose objective was to analyze the processes related to the implementation of an educational innovation supported by the Ley Orgánica de Educación in Spain: the incorporation of basic skills into the educational practices of the centers. The role of educational inspection in such a process is highlighted. The research was conducted using qualitative methodology, through a study of multiple cases in six schools of three different autonomous communities. In those cases, the results show that the role played by education inspectors too often undermines innovation proposals, mainly on the basis of excessive surveillance of documents of the educational centers. The findings lead to a careful reflection on the role that these actors must play in education in the development of public policies, and on the need they adopt a role more focused on counseling. 


\section{LE RÔLE DE L'INSPECTION SCOLAIRE DANS \\ LES PROCESSUS DE CHANGEMENT \\ RÉSUMÉ}

Cet article présente les résultats d'une recherche ayant pour objectif d'analyser les processus liés à la mise en place d'une innovation éducationnelle promue par la Ley Orgánica de Educación en Espagne: l'incorporation des compétences de base aux pratiques éducatives des centres. À cette occasion, le rôle joué par l'inspection éducative dans ce processus est mis en lumière. La recherche a été développée au moyen d'une méthodologie qualitative, basée sur l'étude de cas multiples dans six centres éducationnels de trois communautés autonomes différentes. Les résultats montrent que dans les cas étudiés, le rôle joué par les inspecteurs scolaires représente, très souvent, une entrave aux innovations proposées, surtout en raison d'une surveillance excessive des documents et des centres éducatifs. Les conclusions invitent à une réflexion profonde sur le rôle que ces acteurs éducatifs doivent assumer dans le développement des politiques publiques, et sur la nécessité pour ceux-ci d'adopter un rôle plutôt consultatif.

POLITIQUES ÉDUCATIVES • INSPECTION SCOLAIRE • ÉLABORATION DU CURRICULUM • INNOVATIONS ÉDUCATIVES

\section{O PAPEL DA INSPEÇÃO ESCOLAR NOS PROCESSOS DE MUDANÇA}

RESUMO

Neste artigo são apresentados os resultados de uma pesquisa cujo objetivo foi analisar os processos relacionados com a implementação de uma inovação educacional promovida pela Ley Orgánica de Educación na Espanha: a incorporação das competências básicas às práticas educativas dos centros. Nesta oportunidade destaca-se o papel desempenhado pela inspeção educativa em tal processo. A pesquisa foi realizada mediante uma metodologia qualitativa, realizando um estudo de casos múltiplos em seis centros educacionais de três comunidades autônomas diferentes. Nos casos abordados, os resultados evidenciam que o papel desempenhado pelos inspetores escolares com muita frequência prejudica as inovações propostas, em função, sobretudo, de uma excessiva vigilância dos documentos dos centros. Nesse sentido, as conclusões levam a uma reflexão profunda sobre o papel que esses atores devem assumir no desenvolvimento das políticas públicas e sobre a necessidade de adotarem um papel mais centrado na assessoria. 
El presente artículo se desprende del "Estudio de las dimensiones curriculares, organizativas metodológicas y evaluativas de la competencia socia y ciudadana", el cua ha sido seleccionado y financiado por el Ministerio de Educación y Cultura de España, de acuerdo con la Resolución del 20 de octubre de 2011, de la Secretaría de Estado de Educación y Formación Profesional, por la que se conceden ayudas para los proyectos que profundicen en la consolidación de las competencias básicas como elemento esencial de currículo.
A INVESTIGACIÓN SOBRE LOS PROCESOS DE CAMBIO E INNOVACIÓN EDUCATIVA ha puesto de manifiesto las condiciones que los mismos tienen que asumir para poder avanzar en la dirección prevista (HARGREAVES, 1996; SARASON, 2003; SACRISTÁN, 2006; PUELLES, 2006; VIÑAO, 2006; ANDERSON, 2010; DAY, 2010; FULLAN, 2002, 2010; AGUERRONDO, 2014). Entre estos rasgos es posible destacar los siguientes: a) la importancia de contemplar la dimensión objetiva y subjetiva del cambio de forma simultánea (FULLAN, 2002); b) el cuidado en la formulación de las prescripciones normativas que regulan los cambios (PERRENOUD, 2012); c) la necesidad del trabajo colaborativo y de los procesos participativos en general: del profesorado (MURILLO; KRICHESKY, 2012; MONARCA; SANDOVAL, 2013), de las familias (SIMÓN; ECHEITA, 2012), de los estudiantes (FIELDING, 2012; MESSIOU, 2013); d) la relevancia del "tiempo" como elemento clave en los procesos de cambio e innovación y en las etapas con él relacionadas (ADELMAN; WALKING-EAGLE, 2003; GOODSON, 2010; PERRENOUD, 2012); etc.

En cualquier caso, se ha llamado la atención sobre el verdadero impacto que suelen tener las políticas de reforma y cambio educativo; en esta línea, se ha constatado una gran dificultad "para modificar los patrones de funcionamiento de los sistemas educativos” (TEDESCO, 2012, p. 118), no solo en modalidades centralizadas y fuertemente burocráticas de organización educativa, sino también en estructuras descentralizadas y escasamente burocratizadas. Una de las razones que 
se ofrece para explicar esta dificultad se relaciona con la velocidad y la frecuencia con la que se suceden las políticas de reforma, cambio o innovación. En este sentido, con respecto a la incorporación del enfoque de competencias en España, Bolívar (2008) considera que el profesorado se ha encontrado ante la precipitación y la exigencia de incluir este nuevo elemento curricular sin el tiempo que las evidencias de la investigación aconsejan.

Por su parte, la literatura señala que la gran cantidad de cambios normativos a los que se ha tenido que enfrentar el profesorado en el contexto español ha influido negativamente, generando una cultura, ideas, actitudes, sentimientos, sobre el cambio educativo, que poco ayudan para que este se produzca. Entre estas ideas está la de contemplarlo como una mera exigencia burocrática, sin trascendencia para las prácticas escolares; entre los sentimientos, están la apatía, incredulidad y el agotamiento (ÁLVAREZ, 2008; BOLÍVAR, 2008; DIEZ, 2008). Con respecto a los continuos cambios normativos, Puelles (2006, p. 80) considera que "la continua sucesión de leyes en materias importantes para la sociedad, no sólo produce inseguridad, cansancio, relativismo y escepticismo", sino que además atenta contra el mismo espíritu de la ley. De acuerdo con este autor, para que una ley tenga posibilidad de alcanzar lo que se propone, tiene que conectar con la realidad, para lo cual necesita estabilidad, continuidad, algo que solo es posible, en su opinión, si la ley es "producto de la reunión de los ciudadanos" (PUELLES, 2006, p. 79), es decir, un consenso. Algo en lo que también insisten Hargreaves y Fink (2008), si se pretende que los cambios sean sostenibles, duraderos en el tiempo.

La literatura nos muestra que, con mucha frecuencia, las políticas que procuran introducir innovaciones o cambios educativos suelen poner demasiado énfasis en el nivel político-normativo del cambio, sin atender a una lógica procesual del mismo que llevaría a contemplar, necesariamente, acciones a varios niveles, y a los diversos actores ligados a los mismos (CANTO CHAC, 2008; MONARCA; RAPPOPORT, 2013). Esta lógica de actuación política, caracterizada por una racionalidad técnica, desconoce los múltiples y complejos procesos y dinámicas de configuración de «lo real» (POPKEWITZ; LINDBLAD, 2000; ALMANDOZ, 2010), en los cuales no solo tienen cabida la acción que desarrollan los gobiernos, las administraciones, los partidos políticos y otros grupos de poder más o menos institucionalizados, sino también otros actores, instancias, instituciones, programas y sujetos que resultan imprescindibles, ya no solo vistos como «aplicadores» de las regulaciones normativas que intentan dar direccionalidad a la realidad, sino como verdaderos agentes mediadores, incluso promotores de las mismas (FREIRE; MACEDO, 1989). 
En este sentido, un número reducido de investigaciones ha dado cuenta del papel específico de los inspectores educativos (TYAGI, 2010; RUL, 2013; SILVA, 2013), como actores con un papel especialmente relevante en la mediación entre las pretensiones normativas y su apropiación y desarrollo por parte de los centros educativos. Las evidencias muestran que las características que asume este rol influyen en el desarrollo de procesos de cambio e innovación, según se ubique más próximo al control-supervisión o al asesoramiento (ESCUDERO; MORENO, 2012; AGUERRONDO, 2013), y según se realicen los mismos procesos de control-supervisión.

Este artículo se centra en el papel que ha asumido la inspección educativa en España en el proceso de implementación de las competencias básicas como nuevo componente curricular y nuevo enfoque pedagógico, el cual ha sido promovido y regulado por la Ley Orgánica de Educación (2006).

\section{LA CONFIGURACIÓN DEL ROL DE INSPECCIÓN EDUCATIVA}

La configuración del rol de inspección educativa debe ser abordada desde un análisis multidimensional. Cuando menos es preciso enmarcarlo en una perspectiva histórica, donde la inspección educativa se ha caracterizado fundamentalmente por un papel de control vertical y jerárquico (VIÑAO, 1999; HERNÁNDEZ, 2005), y en los procesos de transformación del Estado y sus mecanismos de gobierno (SANTOS, 2005; AGUERRONDO, 2013), en los que se promueven la descentralización, la desregulación, la privatización y, paradójicamente, se ensayan nuevas formas de control.

Históricamente, el origen de la inspección educativa en España debe ubicarse, tal como propone Ramírez (2003), en el mismo proceso de construcción del Estado moderno, en el paso del Antiguo al Nuevo Régimen, por tanto, en un proceso de ampliación y secularización de la educación. En este sentido, la inspección surge y se ubica en un lugar de mediación entre las políticas, las prescripciones normativas y aquellos que, bajo una lógica tecnocrática, tenían el deber de implementarlas en las instituciones escolares. Estas características se enmarcan en los rasgos de la misma configuración de los estados nacionales y sus sistemas educativos, los cuales se caracterizaron, como expresa Tedesco (2012), por su racionalidad técnico-burocrática. En este contexto, en un sistema educativo organizado jerárquicamente y con una clara función homogeneizadora, la inspección educativa aparece como garante de estas pretensiones en las instituciones escolares. De acuerdo con esa lógica histórica, los gobiernos emplearon con gran frecuencia el uso de la ley como instrumento preferente para cambiar la realidad (TEDESCO, 2012). De esta manera, la configuración del rol de la inspección 
educativa quedó sujeta a esta vigilancia normativa bajo el supuesto de que "la realidad" podía ser construida o modificada a través de la simple aplicación lineal de la misma. Estos postulados acompañaron la instalación de los inspectores en los sistemas educativos y la definición de sus funciones y caracterización de su actuación en los centros escolares.

Tal como sostiene Aguerrondo (2013, p. 16), “este primer sentido de la inspección educativa asociada con la tarea de control, fiscalización administrativa y correcto cumplimiento de las normas y leyes" aún persiste, a pesar de la ampliación actual de sus funciones debido a los cambios sociales que vienen teniendo lugar en las últimas décadas relacionados con: a) las formas de conceptualizar la sociedad; b) las transformaciones en la intervención pública y c) la transformación de las instituciones o desinstitucionalización (DUBET; MARTUCCELLI, 2000). En cuanto a la ampliación de funciones asociadas a este nuevo escenario, Aguerrondo (2013) destaca la implicación en la dimensión pedagógica e institucional, es decir, en los diversos aspectos del funcionamiento del sistema educativo y de los centros escolares. En este contexto se empieza a vincular, cada vez con más insistencia, a la inspección educativa con los procesos de cambio y mejora educativa (FULLAN, 2002; DEDERLING; MÜLLER, 2010; SILVA, 2013), poniendo así en evidencia un escenario de gran complejidad y contradicción en cuanto a las funciones y al lugar específico de este rol dentro de los sistemas educativos modernos. Los aportes de esta investigación sugieren que, para acompañar estos procesos, la inspección educativa debe desarrollar un tipo de práctica centrada en el asesoramiento y la orientación (CANTÓN; VÁSQUEZ, 2010; DOMINGO, 2010).

Esta complejidad queda reflejada en tres tensiones que menciona Viñao (1999): la primera entre politización y profesionalización del cuerpo de inspección; para el autor, es la más significativa de todas, dado que se encuentra muy presente desde el origen de la inspección. La segunda entre la fiscalización-control y el asesoramiento, y la tercera entre lo administrativo y lo pedagógico. De acuerdo con Viñao (1999), estas tensiones siguen de alguna manera presentes, tanto en las prácticas de inspección, supervisión y asesoramiento, como en las representaciones que sobre las mismas y sobre el mismo rol tienen los diversos actores educativos. Sostiene el autor que el modo en que estas tensiones se resuelven determina la configuración de estilos o modelos de inspección.

\section{METODOLOGÍA}

La investigación de la que surge este artículo se desarrolló mediante una metodología cualitativa, realizando un estudio de casos múltiples 
o colectivos, instrumental, de tipo descriptivo-interpretativo (STAKE, 2006). Por otra parte, aunque no es una etnografía en sentido estricto, asumiendo la sugerencia de Wolcott (2006), es preciso reconocer que sí se dan algunas de sus técnicas y procedimientos principales. De esta manera, se reconoce que esta perspectiva ofrece un enorme potencial, tanto epistemológico como metodológico, para aproximarnos a lo social, lo cultural y lo político, desde una visión holística y compleja (VELASCO; DÍAZ DE RADA, 1999).

\section{CASOS}

Teniendo en cuenta la concepción procesual de política y la metodología escogida para la investigación, el caso quedó definido por tres niveles de actuación en lo que respecta a:

1) La objetivación de las políticas, es decir las prescripciones técnico-normativas que definen una pretendida direccionalidad de los sistemas y prácticas educativas.

2) Su concreción en "unidades político-administrativas” específicas, en España denominadas Comunidades Autónomas (CCAA), que tienen, como su nombre lo indica, cierta autonomía, al menos teórica, ${ }^{1}$ a la hora de definir las políticas de su territorio, en este caso tres CCAA: Madrid, Castilla-La Mancha y Castilla y León.

3) Los actores implicados en los procesos de decisión, diseño, apropiación y resignificación de las prescripciones normativas; en este caso, como se ha explicado, se ha puesto el acento en la inspección educativa; sin embargo, en la investigación se ha indagado por lo sucedido I) a nivel macro (agentes que: a) toman decisiones acerca de las políticas, b) las transforman en prescripciones normativas de cualquier estatus normativo, c) desarrollan acciones tendientes a generar las condiciones para el desarrollo de las prescripciones); II) a nivel intermedio (formado por agentes mediadores entre la toma de decisiones y los espacios donde las políticas deben ser desarrolladas-implementadas) y III) a nivel micro (agentes que se ubican en los espacios-instituciones donde las políticas deben tener impactos y producir transformaciones

Esto significa que, aunque normativamente las CCAA tengan las competencias en educación es decir, la posibilidad de asumir decisiones $y$ direccionalidades singulares en el desarrollo de las políticas educativas que fija el Estado, en España esto es llevado a cabo por el conjunto de Comunidades y Ciudades Autónomas; en la práctica, salvo contadas excepciones, no siempre se aprecian grandes diferencias entre ellas. diversas; son agentes que se apropian y resignifican las prescripciones normativas que pretenden dar una direccionalidad a la realidad y, en ese marco, generan determinado tipo de prácticas y acciones); en este caso se han tomado seis instituciones escolares, dos en cada una de las CCAA, una de enseñanza primaria y otra de enseñanza secundaria.

Es preciso aclarar que es un modelo analítico y que los casos se definen en una trama compleja de interacción entre estos niveles. Por otra parte, en esta investigación se ha construido un conocimiento de lo que sucede en estos niveles, principalmente desde "la mirada" de uno de ellos, a través de las representaciones de los actores del nivel micro. 
Esto se ha complementado con el análisis de la normativa y documentos normativos existentes.

\section{INFORMANTES}

En todos los casos los informantes han sido profesores o profesoras de los centros seleccionados, que han sido escogidos de acuerdo con los siguientes criterios: a) antigüedad; b) cargo/función: miembro del equipo directivo, tutor, jefe de departamento, miembro del equipo de orientación o profesor sin ninguno de los cargos/funciones antes mencionadas; c) especialidad; y d) disponibilidad para observación y entrevistas.

\section{CONTEXTOS, PRÁCTICAS Y CONDICIONANTES QUE ATRAVIESAN EL DESARROLLO CURRICULAR EN ESPAÑA}

Teniendo en cuenta que el objeto de indagación de la investigación es una política que procura generar cambio a nivel curricular, se hace necesario analizar aquellos aspectos que contextualizan y actúan como condicionantes de este proceso. En España, el primer nivel de definición curricular le corresponde al Estado, y posteriormente son las Administraciones Educativas quienes deben desarrollar y concretar el currículo. A continuación, en un tercer nivel de concreción, los centros educativos deben adaptar los currículos a sus contextos y desarrollarlos.

De acuerdo con las diversas normativas vigentes, entre ellas la Ley Orgánica de Educación (2006), el Estado, en el primer nivel de concreción de las enseñanzas, define la estructura del sistema educativo y una parte del currículo prescrito. La investigación se ha centrado en la denominada «educación básica», la cual se define como un todo, es decir, como un marco de sentido de la educación y su particular forma de organizarla. Los diez años que la comprenden son los que dan pleno sentido a lo que se entiende por educación básica, común y obligatoria: los contenidos y competencias que se espera que toda la población desarrolle como base de la ciudadanía y del desarrollo personal y social. De acuerdo con la normativa vigente, la educación básica comprende dos etapas: educación primaria y educación secundaria obligatoria. La primera se organiza en tres ciclos, con dos niveles educativos en cada uno de ellos. Se cursa ordinariamente entre los 6 y los 12 años. Por su parte, la educación secundaria obligatoria comprende 4 cursos que se seguirán de forma ordinaria entre los 12 y 16 años de edad. Sin embargo, la edad de permanencia se puede ampliar hasta los 18 años. En cuanto a la organización de los centros educativos, esta es producto de una serie de leyes que se implementaron a partir de 1985, inspiradas en una visión 
democrática y participativa del profesorado en la toma de decisiones. En este sentido, la normativa contempla la existencia de a) órganos de gobierno: consejo escolar, claustro de profesores y equipo directivo, y b) órganos de coordinación docente: comisión de coordinación pedagógica, equipos de ciclo (primaria) o departamentos didácticos (secundaria), departamento de orientación (secundaria), tutores y junta de profesores.

La estructura general del sistema educativo es igual en todo el Estado español; por su parte, de acuerdo con la Ley Orgánica de Educación (2006), el currículo estipulado para cada etapa educativa es definido parcialmente por el gobierno del Estado o Administración Central, términos que se suelen emplear con un mismo sentido. Esta definición central es de un 55\% de los contenidos para las CCAA que tienen lengua cooficial y de un 65\% para aquellas que no la tienen. En cuanto a la investigación desarrollada, las tres CCAA elegidas mantenían una propuesta curricular prácticamente idéntica; en un análisis detallado de la legislación vigente, se ha constatado una escasa variación entre ellas, sin asumir singularidades significativas de acuerdo con ese 35\% de autonomía definida.

En cuanto a la política de cambio específica que se ha abordado para analizar el papel de la inspección educativa en su implementación en las tres CCAA elegidas, se refiere a la incorporación de las competencias básicas como nuevo elemento del currículo y nuevo enfoque pedagógico, regulado normativamente en la Ley Orgánica n. 2/2006, del 3 de mayo, de Educación - LOE -, antes mencionada. Con la misma se inicia una nueva política tendiente a incorporar este enfoque pedagógico a los centros educativos, a las prácticas de enseñanza. Desde el punto de vista de las prescripciones curriculares, más allá de las menciones que hace la LOE a las competencias, tanto el Real Decreto n. 1513/2006 como el Real Decreto n. 1631/2006, por el que se establecen las enseñanzas mínimas de la educación primaria y la educación secundaria obligatoria respectivamente, son los que realmente avanzan en una primera concreción de este componente curricular. Es preciso mencionar que estas decisiones, esta nueva ley de educación, se han enmarcado en una tendencia a la convergencia europea en esta temática y en un discurso en torno a las competencias que asume un rasgo cada vez más globalizado, transnacional (BOLÍVAR, 2008; SARASÚA, 2008; PERRENOUD, 2012). Un antecedente destacable en la aparición e instalación del concepto de competencias clave ha sido el Consejo Europeo celebrado en marzo de 2000 en Lisboa para acordar un nuevo objetivo estratégico de la Unión con el fin de reforzar el empleo, la reforma económica y la cohesión social como parte de una economía basada en el conocimiento. En esta dirección, en el año 2001 los Ministros de Educación establecen un acuerdo sobre los objetivos comunes de educación y formación como meta para el año 2010 (BOLÍVAR, 2008), línea que se continúa 
y profundiza en el Consejo de Europa de Barcelona del año 2002. Fue cobrando forma así la denominada Estrategia de Lisboa, que ha tenido gran influencia en las agendas educativas de los países europeos. En este marco se encuentra la Resolución del Consejo de la Unión Europea del 27 de junio de 2002 sobre la educación permanente (2002/C 163/01, Diario Oficial de las Comunidades Europeas). Hoy las competencias forman parte de los discursos de los organismos internacionales, de las instituciones europeas y de un número importante de países. En este contexto, y asumiendo las recomendaciones de la Comisión y el Parlamento Europeo, la LOE y los Reales Decretos mencionados, de forma similar a como se ha hecho a nivel europeo, incluyen ocho competencias básicas: 1) competencia en comunicación lingüística, 2) competencia matemática, 3) competencia en el conocimiento y la interacción con el mundo físico, 4) tratamiento de la información y competencia digital, 5) competencia social y ciudadana, 6) competencia cultural y artística, 7) competencia para aprender a aprender y 8) autonomía e iniciativa personal. A partir de aquí son las Comunidades Autónomas, de acuerdo a las competencias otorgadas por la constitución y la legislación educativa, las que deben incluirlas en sus respectivos currículos.

En lo que respecta específicamente a la inspección educativa, de acuerdo con la normativa vigente, queda organizada en:

I) Alta Inspección, cuyo sentido queda expresado en el artículo 149 de la LOE de esta forma: "Corresponde al Estado la alta inspección educativa, para garantizar el cumplimento de las facultades que le están atribuidas en materia de enseñanza y la observancia de los principios y normas constitucionales aplicables y demás normas básicas que desarrollan el artículo 27 de la Constitución”. Todas las atribuciones establecidas para la Alta Inspección se centran en funciones de control y vigilancia normativa.

II) Inspección Educativa, según establece la ley antes mencionada, es regulada por las Administraciones educativas de cada CCAA. De las siete funciones que para ella se establecen en el artículo 151 de dicha ley, solo una hace referencia explícita al asesoramiento, aunque en su misma formulación queda limitado su sentido: “Asesorar, orientar e informar a los distintos sectores de la comunidad educativa en el ejercicio de sus derechos y en el cumplimiento de sus obligaciones" (LOE, 2006, art. 151, inciso f).

\section{MODELO DE APROXIMACIÓN AL OBJETO DE ESTUDIO}

De acuerdo con el modelo teórico para el estudio del campo del currículo que es posible derivar de Sacristán (1988), se han contemplado cinco niveles de análisis, los cuales contribuyen a focalizar la atención en aspectos específicos del objeto de estudio: 
1) Nivel I: el currículo prescrito. Constituido por la normativa que fija las competencias como nuevo componente curricular, tanto a nivel estatal como de las Comunidades Autónomas.

2) Nivel II: las acciones desarrolladas por la Administración para incorporar las competencias clave. Contempla todas aquellas acciones y prácticas que la Administración ha puesto en marcha con el objetivo de favorecer la implementación del enfoque de competencias. En este caso, entre otras cosas, se focaliza la atención en el papel de la inspección educativa, si es que lo ha tenido.

3) Nivel III: las acciones desarrolladas por los centros para incorporar las competencias clave. Se refiere a las acciones centradas en la elaboración de los documentos del centro, a los documentos en sí mismos y a cualquier otro tipo de acción o práctica relacionada con la implementación de las competencias. En este nivel se aborda también, de forma específica, el papel que juega la inspección en las acciones mencionadas.

4) Nivel IV: el currículo en acción. Comprende las acciones, programas y proyectos desarrollados por el profesorado y el centro. Con respecto a la inspección educativa, se pone el acento en la forma en que la inspección educativa interviene en este nivel o en los efectos que ella tiene en el mismo.

5) Nivel V: currículo evaluado. Se refiere a las prácticas y acciones de evaluación de las competencias. De la misma manera que en los casos anteriores, se ha indagado el papel de la inspección educativa en relación a este nivel.

\section{TÉCNICAS Y PROCEDIMIENTOS}

De acuerdo con la opción metodológica realizada, se han empleado las siguientes técnicas de investigación: A) entrevistas, un total de 76. De ellas 15 han sido holísticas, es decir, destinadas a suministrar contexto (VELASCO; DÍAZ DE RADA, 1999), y las 61 entrevistas restantes han sido focalizadas en el objeto de estudio. B) Observaciones descriptivas/abiertas y focalizadas/selectivas; algunas de ellas han sido participativas. C) Análisis de documentos. Se han contemplado dos tipos principales de documentos:

1. Prescriptivos: I) Nivel estatal: a) LOE; b) Real Decreto n. 1513/2006, del 7 de diciembre, por el que se establecen las enseñanzas mínimas de la educación primaria; y c) Real Decreto n. 1631/2006, del 29 de diciembre, por el que se establecen las enseñanzas mínimas correspondientes a la educación secundaria obligatoria. II) Nivel autonómico: la normativa específica de cada una de las Comunidades Autónomas escogidas.

2. Elaborados por el profesorado: a) Proyecto educativo de centro PEC; b) Programación general anual - PGA; c) Plan de atención a la 
diversidad (PAD); d) Plan de acción tutorial - PAT; e) Programaciones didácticas; y f) otros planes o programas, según los casos: planes de convivencia, plan de diversificación curricular, memorias anuales, etc.

\section{TRABAJO DE CAMPO}

El trabajo de campo se ha desarrollado a lo largo de un curso escolar completo. El mismo se ha dividido en dos fases específicas y un tercer momento de devolución. La idea fue iniciar un proceso de aproximación progresiva a nuestro objeto de investigación a través de las dos primeras fases mencionadas. La fase 1, considerada conceptual y metodológicamente como una aproximación holística (VELASCO; DÍAZ DE RADA, 1999), ha tenido tres objetivos: a) construir esta visión holística para identificar posibles “ámbitos” o “áreas” de indagación más específicas; b) identificar posibles informantes, personas relevantes desde el punto de vista del discurso específico relacionado con el objeto de estudio; y c) construir una relación de confianza y cercanía, imprescindible para el acceso a la cultura. La fase 2, de profundización y focalización, se ha centrado en los objetivos específicos de la investigación. Es preciso aclarar que estas fases no siempre se pueden ver como claramente diferenciables. Los límites entre las mismas son difusos y ellas han funcionado fundamentalmente como organizadoras de intenciones y acciones relacionadas con el trabajo de campo. Sin embargo, a lo largo del desarrollo de este trabajo, aunque a grandes rasgos se ha mantenido la estructura de fases mencionada, se han dado circunstancias que han variado esta estructura. Lo importante no es la estructura de fases como algo sincrónico a seguir, sino desde el punto de vista de los objetivos que cada una de ellas tiene.

\section{INSPECCIÓN EDUCATIVA, NORMATIVA, REALIDAD Y BUROCRATIZACIÓN DE LOS PROCESOS DE CAMBIO}

De acuerdo con las representaciones de los informantes entrevistados, un rasgo que parece predominar sobre la actuación de la inspección educativa es su papel centrado predominantemente en la supervisión y seguimiento de la implementación de la normativa, su aplicación. Aquí aparece nuevamente una lógica que, de acuerdo con los relatos, parece funcionar solo desde el punto de vista burocrático, entendido en este caso, y de acuerdo con los aportes de Barroso (2005), en dos sentidos: como prerregulación y como regulación institucional (normativa y de control); se trata de un modo de ajustar la acción a priori, incluso ajena, a los procedimientos que se implementan para obtener determinados resultados. Un aspecto sorprendente que aparece en los relatos es que esta forma de ejercer la supervisión asume un supuesto de importantes 
consecuencias; la presencia de la normativa en los documentos de los centros educativos parece entenderse como la presencia en la misma realidad, en las prácticas escolares. Se da por supuesto que, si los documentos reflejan el cambio que la norma propone, las prácticas escolares también lo hacen. En cualquier caso, los elementos que se han podido recoger en el trabajo de campo, ya sea en las entrevistas, observaciones o en los documentos analizados, no permiten concluir hasta qué punto esta situación es producto de asumir de forma explícita este supuesto o si, en cambio, esta forma de prerregulación o de regulación institucional (BARROSO, 2005) es producto de las mismas contradicciones en las que se ubican actualmente las múltiples demandas de la inspección educativa, junto con las limitaciones que se encuentran para desarrollar algunas de ellas.

En cualquier caso, tal como nos informan los entrevistados, si la supervisión se centra en «la aplicación», y si esta se constata exclusivamente, mediante su presencia, en muchos casos literal, en los documentos del centro, se traslada una idea muy particular sobre cómo se configura la realidad educativa o, siendo más preciso, configura dos contextos de realización que, de acuerdo con el trabajo de campo, terminan estando escindidos, generando dos realidades o prácticas con lógicas diferenciadas, las cuales suponen actuaciones específicas y muchas veces desconectadas: las prácticas y racionalidad que giran en torno al diseño de documentos, y las prácticas y racionalidad que giran en torno al trabajo del aula o al desarrollo del currículo, al currículo en acción. En este sentido, una actuación de la inspección que toma como centro de su actuación los documentos de los centros educativos contribuye a transformarlos en una práctica con un valor en sí mismo y sin necesaria conexión con la práctica educativa. En concreto, se ha apreciado que el profesorado, bajo esta lógica de actuación, deba dedicar importantes esfuerzos a esta tarea de elaboración de documentos. En este mismo sentido, de acuerdo con lo que se ha recogido en los relatos de los profesores, estos procesos de supervisión centrados en la «vigilancia de la normativa» focalizada en los documentos del centro, no parecen aportar nada a la transformación de las prácticas educativas en sí mismas. El cambio, según las evidencias de la investigación (FULLAN, 2002; ADELMAN; WALKING-EAGLE, 2003; HARGREAVES; FINK, 2008), está más asociado a procesos de búsqueda, indagación, confianza, formación, asesoramiento, colaboración, que a procesos de vigilancia, la cual genera desconfianzas y temores, y esto se suma a los que ya de por sí pueden incitar cualquier cambio. Sin embargo, en los casos analizados, se ha comprobado solo una función de inspección educativa basada, como se ha dicho, en la prerregulación o control institucional, ejercido, por otra parte, casi exclusivamente a través de diversos tipos de documentos. 
Algo que se ha podido constatar, tanto en primaria como en secundaria, es que estas prácticas de vigilancia de la norma, centradas en su presencia o no en los documentos de centro, refuerzan la burocratización del cambio, entendido esto como el fomento de prácticas basadas en un control a priori, incluso independiente, de las prácticas educativas. En este sentido, por burocratización se está haciendo referencia a que el cambio que las políticas pretenden se transforma en un proceso estático, principalmente, y en ocasiones exclusivamente orientado a la modificación de documentos.

Por otra parte, a la luz de los relatos, se aprecia que esta lógica de actuación de la inspección educativa, ese excesivo cuidado en que la ley aparezca claramente reflejada en los documentos de los centros educativos, tiene consecuencias en las prácticas que giran en torno a su elaboración. En este sentido, se ha podido constatar que, de acuerdo con los entrevistados y, sobre todo, con el análisis de los documentos, para ser en esta pretensión de la inspección educativa, es copiando los que la misma ley prescribe. Este hecho, que la norma aparezca fielmente reflejada en los documentos de centro (copiada) es algo que, por otra parte, y tal como se mencionó anteriormente, se aprecia también en las leyes que corresponden a distintos niveles de concreción, que se repiten una tras otra, más allá de su estatus normativo, aportando pocas novedades con respecto a la anterior, sin reflejar un proceso gradual de concreción, tal como es de esperar.

La práctica de ciertos inspectores, tal como confirman algunos de los entrevistados, basada en una «lista de control» en la que se «señala» la presencia o no de ciertos «elementos normativos» en las programaciones y otros documentos de los centros educativos como prueba de su existencia, va generando una dinámica de implementación de los cambios, excesiva y artificialmente centrada en esta «lógica formal» que no tiene, necesariamente, tal como se puede constatar, ningún correlato con las prácticas educativas que desarrollan el currículo. La dinámica que se va generando basada en esta lógica formal, de prerregulación o de control institucional (BARROSO, 2005), se puede resolver de forma relativamente sencilla, basta con incluir estos elementos, con trasladarlos «literalmente de la norma a los documentos», algo que en parte ha quedado de manifiesto en los análisis que se han realizado de los mismos.

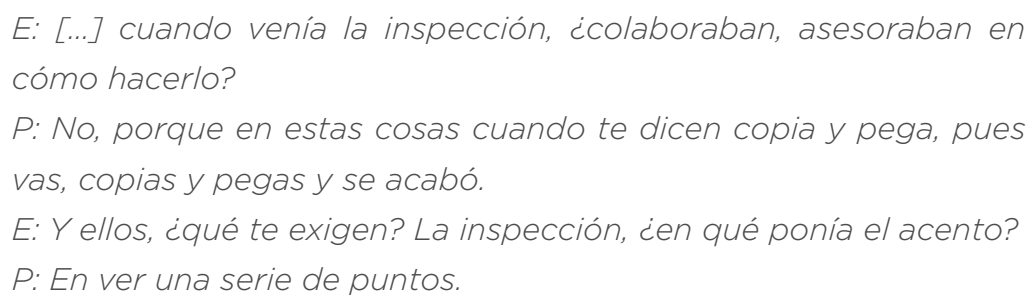




\begin{abstract}
E: ¿En los documentos?
P: Claro, entonces te dicen, a ver estos puntos, ¿están?, si o no, si, pues ya está [...] Los inspectores te dicen, la ley dice esto, aplícalo; digo, pero es que esto contradice, ya, pero es lo que dice la ley [...] Lo que pasa, es que ellos resuelven en el artículo [...] Eso está en su papel [...]. (JorgeCYLESO6P22)
\end{abstract}

Esta "copia literal de la norma” parece ser eficaz a esta lógica formal que caracteriza la actuación de la inspección educativa; al menos, por lo que se deduce de los relatos, de momento les está funcionando, cubre las expectativas de la inspección, sus demandas. Algo que, como es evidente, no ofrece ninguna información de lo que efectivamente está pasando. Tal como explicaba uno de los entrevistados, si la acción del inspector solo se basa en la normativa, es decir, si no ofrece ninguna transformación de la misma, si no ofrece nada nuevo y distinto a lo que ella dice, en realidad, no se ofrece nada que necesiten los centros.

En lo que se refiere específicamente a la política de cambio que se estaba analizando -la incorporación de un nuevo elemento curricular y un nuevo enfoque pedagógico centrado en las competencias básicas-, la ley no es clara para el profesorado, las diversas lógicas contenidas generan más confusión que otra cosa a los docentes. De su lectura no se deduce, al parecer, nada que haga referencia a «cómo hacerlo», ni como centro ni como docente. En este sentido, si los inspectores no asumen ningún papel en esta tarea, su presencia queda reducida a supervisar un proceso que el profesorado no termina de comprender: ni lo que realmente pretende ni lo que se espera de él. Por tanto, ¿qué sentido tiene esta supervisión para la mejora educativa? De acuerdo con los relatos de los profesores, lo que no se sabe hacer es, precisamente, aplicar una norma tan poco clara en esta temática.

[...] la inspección se dedica más bien a ver si te falta el punto 1.3/1 en la programación. (HoracioCYLESO6P12)

E: ¿Crees que esta forma de actuar refuerza la burocratización de las cosas?

J: Si, lo creo, y también creo que es una sensación que tiene todo el profesorado, que hay mucha burocracia, que la Administración se centra en papeles y ya está [...] Cuando viene el inspector, se centra en unas cuantas cosas, la mayoría, por no decir todas, son papeles, documentos. Si ahi está lo que él considera, más allá de si se hace o no, y cómo se hace, ya está. (SandraCAMESO4ED4)

Esta opinión, bastante generalizada en nuestros relatos, según la cual a la Administración le interesa más “el papel”, los documentos, que 
aquello que realmente está sucediendo en las aulas, es muy ilustrativa de la lógica que se ha mencionado y los procesos de burocratización (prerregulación o control institucional) que se refuerzan con este tipo de demandas:

[...] yo te digo que en el ciclo, cuando hacemos, rellenamos un papel, intentamos reflejar la realidad, pero por ejemplo, la administración no tiene en cuenta la realidad, le da igual, la administración solo ve el papel. (DanielCYLEP5P5)

\section{LOS EFECTOS DE UNA ACTUACIÓN BASADA EN EL CONTROL INSTITUCIONAL O PRERREGULACIÓN EN LA CONFIGURACIÓN DE LAS PRÁCTICAS EDUCATIVAS}

Esta práctica de la inspección educativa basada en lo que se ha denominado «lógica formal» (prerregulación o control institucional), teniendo en cuenta que al parecer está muy instalada en la cultura de los centros, está teniendo otros efectos. Para cumplir con esta lógica, no se precisa de ninguna instancia de debate, deliberación, interrogación; siendo claro el producto esperado, cualquier otra cosa, como las anteriores, solo puede desviar la atención del objetivo principal: «que la ley quede reflejada sin lugar a dudas». Las dudas reales de los docentes, las incertidumbres, los interrogantes que puede traer aparejado cualquier cambio, deben ser aparcados, al menos en cuanto a la elaboración de documentos se refiere; en ellos se pide, se buscará, la presencia de la ley. Para acometer esta tarea, por tanto, no hace falta debate, este puede generar más dudas, dilemas o visiones alternativas que no tienen cabida en esta lógica formal mencionada; hace falta que una persona se ponga a transcribir, tarea que suele recaer en una persona concreta, no en un equipo de ciclo ni en un departamento. Dicho esto, es necesario afirmar que, aunque esta parece ser la práctica dominante, eso no quita que se haya podido acceder a documentos que se presentan como innovadores, o con un aporte colectivo significativo. Tampoco quita que en ocasiones sí hayan tenido lugar debates e instancias de intercambio de mayor o menor intensidad y de mayor o menor relevancia, ya que sí se ha podido constatar su existencia. Lo que sucede es que muchas veces aparecen como prácticas no vinculadas, los debates por un lado y el diseño de documentos por otro.

En cualquier caso, es necesario prestar atención a este aspecto que ha aparecido de forma reiterada en los relatos de los profesores; esta práctica de la inspección parece estar jugando un papel clave en la burocratización de las políticas, es decir, que se quede detenida en esta instancia de prerregulación. En este sentido, la formalización 
de las mismas en meros diseños de documentos se transforma en un obstáculo en su desarrollo real, en su llegada a las aulas.

\begin{abstract}
Yo vivo todo como una especie de farsa, o sea, en realidad (lo que hace) es el control de lo que nosotros ponemos en papel. Pero yo creo que, al final, hay un distanciamiento de lo que es la práctica, el día a día. (InésCAMESO4P10)
\end{abstract}

Siempre se ha sentido como una imposición de la inspección, que te hacía cumplir unos items en la programación y entonces te obligaba, digamos, a cruzar lo que tú programas con los items que te estaba exigiendo en esa programación. (MarceloCAMESO4P16)

Los inspectores son unos señores que no vienen más que a ver papeles ahora. Ahora, pero antes no [...] Tuve una inspectora, mayor para mi, no creo que fuese mayor que yo ahora, ni mucho menos, pero yo entonces tenía 25 años, y era muy mayor para mí. Encantadora, fabulosa. A mí, llegó a mi clase, y me dijo qué bien tienes este rincón, de lo que fuese, pero mira, esto no lo trabajas bien. Te puedo decir que esto lo puedes trabajar mejor de esta manera [...] Esa mujer era una inspectora, y lo que hay ahora no son inspectores. No son más que unos señores que vienen a sellarte lo que es válido y lo que no es válido. (CarmenCYLEP5P33)

Esta última profesora valora justamente de la inspección, a partir de su experiencia pasada, el asesoramiento que ha recibido en un momento en el que justamente se iniciaba como profesora. Valora enormemente dos cosas, que la inspectora entrara en su aula y que le ofreciera un consejo sobre cómo mejorar lo que estaba haciendo; algo que para esta profesora parece ofrecerle legitimidad a este rol: conocer la realidad y saber hacer lo que demanda a los profesores. Esta función asesora por parte de la inspección (DOMINGO, 2010), es la que al parecer no se visualiza por la mayoría de los informantes; en cambio se cuestiona, critica, la función que se percibe con más frecuencia, la de supervisar por medio del control de los documentos del centro.

En cambio, el profesorado valora el papel de aquellos inspectores que contribuyen en la implementación que la Administración exige o demanda, mediante claros procesos de asesoramiento y apoyo, en este caso relacionados con el enfoque de competencias. También se valora cuando los inspectores interactúan no solo con el director, sino también con los jefes de departamento; ya que al parecer el principal vínculo que establecen es con el/la director/a, jefes de estudio y jefes de departamento; algo que también hay que ubicar en las condiciones de 
trabajo que deben desempeñar, entre otras, la cantidad de centros a los que deben supervisar-asesorar.

No hay que dejar de advertir que en la misma configuración del rol de inspección, intervienen también las expectativas y representaciones que tienen directores y profesorado en general sobre el mismo. En este sentido, aparece la idea, la presencia, de necesidad de un control de lo que se hace, junto con las demás demandas, expectativas y representaciones que se depositan en él, configurando un rol híbrido.

\section{CONCLUSIONES}

Se aprecia con claridad que, al igual que muchas veces se habla de la Administración o los políticos como algo alejado de los centros, a la inspección educativa se la percibe como totalmente alejada o ausente de las aulas. Aunque se asuma que dentro de sus funciones la supervisión y el control puedan resultar necesarios, no parece entenderse cómo puede realizarse esta tarea solo a partir de la vigilancia normativa a través de la lectura y revisión de documentos del centro. A partir de los relatos recogidos se ha formulado esta interpretación a modo de hipótesis explicativa: el tomar estos documentos como la realidad en sí misma, como si fueran las prácticas educativas en sí, hace que las políticas queden estancadas en esa fase, la cual se resuelve, en muchas ocasiones, con un «recorto y pego». Lo que dice la normativa pasa directamente a los documentos, muchas veces sin «mediaciones» de ningún tipo, sin reflexión, sin debate, sin ajustes, reproduciéndose así la misma lógica que se aprecia en las distintas normativas que reproducen una vez tras otra lo que dice la norma de rango superior. Si esto es dado por bueno, es decir, si la presencia de «la novedad» en el documento se asume como suficiente evidencia del cambio, entonces ahí se paraliza el proceso. Si en esto, como aparece en los relatos de los informantes, es donde se detienen una y otra vez los inspectores, exigiendo modificaciones solo en esta dirección, en los documentos, el proceso se burocratiza, se queda en la fase de prerregulación, previo al desarrollo de aquellas prácticas o acciones que pudiesen buscar el desarrollo del cambio normativamente pretendido. Todo el esfuerzo pasa a centrarse en hacer los mejores documentos posibles, en lo cual no parece haber margen para la confusión, para el error, para la duda... se eliminan por tanto los procesos que pueden demorar esta concreción: debates, consultas, incluso que se plasme algo que no encaje perfectamente con lo que la normativa dice, o que haya alguna duda al respecto.

En cualquier caso, esta práctica, al parecer frecuente de la inspección educativa, esta lógica de actuación, debe enmarcarse en un escenario educativo y social más amplio y complejo, en el cual la forma en que se representa lo social y las instituciones ha sufrido 
un cambio profundo. Tal como sugieren Dubet y Martuccelli (2000, p. 201), "ninguna de estas instituciones funciona según el modelo clásico, como aparatos capaces de transformar valores en normas, y las normas en personalidades individuales”. De acuerdo con esta perspectiva, la sociedad, el sistema educativo y la escuela para la que fue creado el rol de inspección educativa, ya no son los mismos. El proceso de desinstitucionalización que hacen estos autores pone en cuestión los objetivos, las funciones, los roles de la escuela y sus profesionales. En este contexto, como se ha podido apreciar, la configuración del rol de inspección resulta de un cruce complejo de demandas y expectativas, tanto aquellas que fija la normativa, las que cada Administración realiza en un momento concreto de su gestión y de acuerdo con los vaivenes y cambios de gobierno y aquellas que los mismos directores y profesorado realizan en general; un contexto de demandas más versátil, menos claro, menos evidente.

La hipótesis anticipada, de que las formas que asumen los mismos procesos de control, en este caso mediante las funciones asignadas a la inspección educativa, pudiesen explicar parte de la no implementación de las pretensiones de los cambios normativamente estipulados, debe ser profundizada mediante nuevas investigaciones. Por otra parte, esta hipótesis podría ser acompañada, complementariamente, de otras dos hipótesis, aunque en este caso, no se desprenden de la presente investigación. Las mismas surgen de las evidencias de los cambios que se están produciendo en la organización y gestión de Estado (SANTOS, 2005). Hay coincidencias en la existencia de nuevos mecanismos de control externo, enmarcadas por algunos autores en las denominadas formas posburocráticas de control (BARROSO, 2005), con una importante influencia de organismos supranacionales (TARABINI; BONAL, 2011; VALLE, 2013). Así, una de estas primeras hipótesis complementarias es que, en este nuevo contexto, son otros los mecanismos y formas que ejercen control; en este contexto, el rol de la inspección queda descolocado y refugiado en sus antiguas funciones. Sin embargo, a colación, formulamos una segunda hipótesis complementaria; la misma se desprende de la concepción weberiana de burocracia en la que esta se vincula con el control del cumplimiento de los objetivos legítimamente fijados por el Estado. En este sentido, si este control no se está ejerciendo, es decir, si no se comprueba, evalúa, acompaña el impacto de estos objetivos en la transformación de las realidades, se establece una importante fractura en el desarrollo de las políticas que, por otra parte, deja el terreno libre para las otras formas y mecanismos de control mencionados. De esta manera, se podría concluir que el conocimiento construido en el marco de esta investigación permite apreciar la emergencia de una nueva forma de burocracia de control, en la que aún la inspección no tiene un lugar claro. 


\section{REFERENCIAS}

ADELMAN, Nancy; WALKING-EAGLE, Karen. Los docentes, el tiempo y la reforma escolar. In: HARGREAVES, A. (Comp.). Replantear el cambio educativo: un enfoque renovador. Buenos Aires: Amorrortu, 2003. p. 138-162.

AGUERRONDO, Inés. El rol de la supervisión educativa en la gestión de las políticas públicas. Educar, v. 49, n. 1, p. 13-27, 2013.

AGUERRONDO, Inés. Planificación educativa y complejidad: gestión de las reformas educativas. Cadernos de Pesquisa, São Paulo, v. 44, n. 153, p. 548-578, jul./set. 2014.

ALMANDOZ, María Rosa. Las lógicas de las decisiones políticas en educación. In: FRIGERIO, G.; DIKER, G. (Comp.). Educar: ese acto político. Paraná, AR: La Hendija, 2010. p. 53-61.

ÁLVAREZ, Juan Manuel. Evaluar el aprendizaje en una enseñanza centrada en competencias. In: SACRISTÁN, J. Gimeno (Comp.). Educar por competencias, ¿qué hay de nuevo? Madrid: Morata, 2008. p. 206-233.

ANDERSON, Gary. Nuevas "grandes preguntas» sobre el cambio educativo en la siguiente década. Revista Mexicana de Investigación Educativa, v. 15, n. 47, p. 1117-1123, 2010.

BARROSO, João. O estado, a educação e a regulação das políticas públicas. Educação \& Sociedade, Campinas, v. 26, n. 92, p. 725-751, 2005.

BOLÍVAR, Antonio. Ciudadanía y competencias básicas. Sevilla: Fundación ECOEM, 2008.

CANTO CHAC, Manuel. Gobernanza y participación ciudadana en las políticas públicas frente al reto del desarrollo. Política y Cultura, n. 24, p. 9-37, 2008.

CANTÓN, Isabel; VÁSQUEZ, José Luis. Los procesos en gestión de calidad. Un ejemplo en un centro educativo. Revista Iberoamericana sobre Calidad, Eficacia y Cambio en Educación, v. 8, n. 5, p. 59-68, 2010.

DAY, Christopher. El futuro de la investigación en contextos de cambio educativo. Revista Mexicana de Investigación Educativa, v. 15, n. 47, p. 1131-1138, 2010.

DEDERLING, Kathrin; MÜLLER, Sabine. School improvement through inspections? First empirical insights from Germany. Journal of Educational Change, v. 12, n. 3, p. 301-322, 2010.

DIEZ, Carlos La competencia social y ciudadana en la LOE. Avances en Supervisión Educativa, n. 9, 2008. Disponível em: <www.adide.org/revista/index.php?option=com_content\&task=view\&id= 424\&Itemid=65>. Acesso em: dez. 2013.

DOMINGO, Jesús. Comprender y redireccionar las prácticas de asesoramiento. Revista Iberoamericana de Educación, n. 54, p. 65-83, 2010.

DUBET, François; MARTUCCELLI, Danilo. ¿En qué sociedad vivimos? Buenos Aires: Losada, 2000.

ESCUDERO, Juan Manuel; MORENO, María de los Ángeles. Mejorar la educación, la autonomía de los centros y el servicio de inspección educativa. Avances en Supervisión Educativa, n. 17, 2012. Disponível em: <www.adide.org/revista/index.php?option=com_content\&task=view\&id=469\&It emid=73>. . Acesso em: dez. 2013.

FIELDING, Michael. Beyond Student Voice: Patterns of Partnership and the Demands of Deep Democracy. Revista de Educación, n. 359, p. 45-65, 2012.

FREIRE, Paulo; MACEDO Donaldo. Alfabetización: lectura de la palabra y lectura de la realidad. Barcelona: Paidós, 1989.

FULLAN, Michael. Los nuevos significados del cambio en la educación. Barcelona: Octaedro, 2002.

FULLAN, Michael. Investigación sobre el cambio educativo: presente y futuro. Revista Mexicana de Investigación Educativa, v. 15, n. 47, p. 1100-1106, 2010.

GOODSON, Ivor. Teorías del cambio educativo y los contextos históricos. Revista Mexicana de Investigación Educativa, v. 15, n. 47, p. 1139-1145, 2010.

HARGREAVES, Andy. Profesorado, cultura y postmodernidad: cambian los tiempos, cambia el profesorado). Madrid: Morata, 1996. 
HARGREAVES, Andy; FINK, Dean. El liderazgo sostenible: siete principios para el liderazgo en centros educativos innovadores. Madrid: Morata, 2008.

HERNÁNDEZ, Víctor. Asesorando a centros educativos implicados en procesos de mejora escolar. Revista Electrónica Iberoamericana de Calidad, Eficacia y Cambio en Educación, v. 3, n. 1, p. 494-502, 2005.

MESSIOU, Kyriaki. Engaging with students' voices: using a framework for addressing marginalisation in schools. Revista de Investigación en Educación, v. 3, n. 11, p. 86-96, 2013.

MONARCA, Héctor; RAPPOPORT, Soledad. Investigación sobre los procesos de cambio educativo: el caso de las competencias básicas en España. Revista de Educación, n. extraordinario, p. 54-78, 2013.

MONARCA, Héctor; SANDOVAL, Marta. Profesores que se apoyan frente a la complejidad del proceso de inclusión educativa. In: ECHEITA, G. et al. Cómo fomentar las redes naturales de apoyo en el marco de una escuela inclusiva: propuestas prácticas. Sevilla: Eduforma, 2013. p. 77-99.

MURILLO, Francisco Javier; KRICHESKY, Gabriela. El proceso del cambio escolar: una guía para impulsar y sostener la mejora de las escuelas. Revista Iberoamericana sobre Calidad, Eficacia y Cambio en Educación, v. 10, n. 1, p. 26-43, 2012.

PERRENOUD, Philippe. Cuando la escuela pretende preparar para la vida. ¿Desarrollar competencias o enseñar otros saberes? Barcelona: Graó, 2012.

POPKEWITZ, Thomas; LINDBLAD, Sverker. Educational governance and social inclusion and exclusion: some conceptual dificulties and problematics in policy and research. Discourse: Studies in the Cultural Politics of Education, v. 21, n. 1, p. 5-44, 2000.

PUELLES, Manuel. Los hitos reformistas: la viabilidad de las reformas y la perversión de las leyes. In: GIMENO, J. (Comp.). La reforma necesaria: entre la política educativa y la práctica escolar. Madrid: Morata, 2006. p. 61-80.

RAMÍREZ, Elías. Educación y control en los orígenes de la España liberal. Madrid: Biblioteca Nueva, 2003.

RUL, Jesús. Problemas y retos de la inspección de educación. Educar, v. 49, n. 1, p. 29-48, 2013.

SACRISTÁN, J. Gimeno. El curriculum: una reflexión sobre la práctica. Madrid: Morata, 1988.

SACRISTÁN, J. Gimeno. De las reformas como política a las políticas de reforma. In:

SACRISTÁN, J. Gimeno (Comp.). La reforma necesaria: entre la política educativa y la práctica escolar. Madrid: Morata, 2006. p. 23-42.

SANTOS, Boaventura de Sousa. Reinventar la democracia, reinventar el Estado. Buenos Aires: Flacso, 2005. Disponível em: <http://bibliotecavirtual.clacso.org.ar/clacso/se/20100613090848/ reinventar.pdf $\geq$. Acesso em: dez. 2014.

SARASON, Seymour. El predecible fracaso de la reforma educativa. Barcelona: Octaedro, 2003.

SARASÚA, Avelino. Educación para la ciudadanía y de los derechos humanos. Avances en Supervisión Educativa, n. 9, 2008. Disponível em: <www.adide.org/revista/index. php?option=com_content\&task=view\&id=421\&Itemid=65>. Acesso em: dez. 2013.

SILVA, Blanca. El papel de la inspección escolar en la mejora de los resultados educativos. Educar, v. 49, n. 1, p. 67-82, 2013.

SIMÓN, Cecilia; ECHEITA, Gerardo. La alianza entre las familias y la escuela en la educación del alumnado más vulnerable. Padres y Maestros, n. 344, p. 31-34, 2012.

STAKE, Robert. Evaluación comprensiva y evaluación basada en estándares. Barcelona: Graó, 2006.

TARABINI, Aina; BONAL, Xavier. Globalización y política educativa: los mecanismos como método de estudio. Revista de Educación, n. 355, p. 235-255, 2011.

TEDESCO, Juan Carlos. Educación y justicia social en América Latina. Buenos Aires: Fondo de Cultura Económica, Universidad Nacional de San Martín, 2012.

TYAGI, Rajvir Singh. School-based. Instructional supervision and the effective professional development of teachers. Journal of Comparative and International Education, v. 40, n. 1, p. 11-125, 2010. 
VALLE, Javier. Supranational education: a new field of knowledge to address educational policies in a global world. Journal of Supranational Policies of Education, n. 1, p. 7-30, 2013.

VELASCO, Honorio; DÍAZ DE RADA, Ángel. La lógica de la investigación etnográfica: un modelo para etnógrafos de la escuela. Valladolid: Trotta, 1999.

VIÑAO, Antonio. El éxito o fracaso de las reformas educativas: condicionantes, limitaciones, posibilidades. In: SACRISTÁN, J. Gimeno (Comp.). La reforma necesaria: entre la política educativa y la práctica escolar. Madrid: Morata, 2006. p. 43-60.

WOLCOTT, Harry. Sobre la intención etnográfica. In: VELASCO, H.; GARCÍA, F. J.; DÍAZ DE RADA, Á. (Ed.). Lecturas de antropología para educadores. Madrid: Trotta, 2006. p. 127-144.

\section{REFERENCIAS LEGALES}

DIARIO OFICIAL DE LA UNIÓN EUROPEA (DOUE) (2006). Competencias clave para el aprendizaje permanente - Un marco europeo es el anexo de una Recomendación del Parlamento Europeo y del Consejo de 18 de diciembre de 2006 sobre las competencias clave para el aprendizaje permanente (2006/962/CE). Disponível en: <http://eur-lex.europa.eu/ LexUriServ/site/es/oj/2006/1_394/1_39420061230es00100018.pdf>. Acesso em: mar. 2012.

ESPAÑA. Ministerio de Educación y Ciencia. LEY ORGÁNICA 2/2006, de 3 de mayo, de Educación. Boletín Oficial del Estado (B.O.E), n. 106, de jueves 4 mayo 2006.

ESPAÑA. Ministerio de Educación y Ciencia. REAL DECRETO 1513/2006, de 7 de diciembre, por el que se establecen las enseñanzas mínimas de la educación primaria. Boletín Oficial del Estado (BOE), n. 293, del 8 de diciembre de 2006.

ESPAÑA. Ministerio de Educación y Ciencia. REAL DECRETO 1631/2006, de 29 de diciembre, por el que se establecen las enseñanzas mínimas correspondientes a la educación secundaria obligatoria. Boletín Oficial del Estado (BOE), n. 5, del 5 de enero de 2007.

\section{HÉCTOR MONARCA}

Profesor de la Universidad Autónoma de Madrid, Madrid, Espanha;

Secretario Académico del Departamento de Didáctica y Teoría de la Educación; miembro del Grupo de Investigación sobre "Políticas Educativas Supranacionales" - GIPES -, Madrid, Espanha

hector.monarca@uam.es

\section{NOELIA FERNÁNDEZ-GONZÁLEZ}

Doctoranda del Programa de Educación de la Facultad de Formación de Profesorado y Educación de la Universidad Autónoma de Madrid, Madrid, Espanha nfg.fernandeznoelia@gmail.com 